\title{
Enhanced Higgs associated production with a top quark pair in the NMSSM with light singlets
}

\author{
Marcin Badziak $^{a, b}$ and Carlos E.M. Wagner ${ }^{c, d, e}$ \\ ${ }^{a}$ Institute of Theoretical Physics, Faculty of Physics, University of Warsaw, \\ ul. Pasteura 5, PL-02-093 Warsaw, Poland \\ ${ }^{b}$ Berkeley Center for Theoretical Physics, Department of Physics and \\ Theoretical Physics Group, Lawrence Berkeley National Laboratory, University of California, \\ Berkeley, CA 94720, U.S.A. \\ ${ }^{c}$ Enrico Fermi Institute, University of Chicago, \\ Chicago, IL 6063\%, U.S.A. \\ ${ }^{d}$ High Energy Physics Division, Argonne National Laboratory, \\ Argonne, IL 60439, U.S.A. \\ ${ }^{e}$ Kavli Institute for Cosmological Physics, University of Chicago, \\ Chicago, IL 6063\%, U.S.A. \\ E-mail: mbadziak@fuw.edu.pl, cwagner@hep.anl.gov
}

Abstract: Precision measurements of the $125 \mathrm{GeV}$ Higgs resonance recently discovered at the LHC have determined that its properties are similar to the ones of the Standard Model (SM) Higgs boson. However, the current uncertainties in the determination of the Higgs boson couplings leave room for significant deviations from the SM expectations. In fact, if one assumes no correlation between the top-quark and gluon couplings to the Higgs, the current global fit to the Higgs data lead to central values of the Higgs couplings to the bottom-quark and the top-quark that are about $2 \sigma$ away from the SM predictions. In a previous work, we showed that such a scenario could be realized in the Next to Minimal Supersymmetric extension of the SM (NMSSM), for heavy singlets and light MSSM-like Higgs bosons and scalar top quarks, but for couplings that ruined the perturbative consistency of the theory up to the GUT scale. In this work we show that a perturbative consistent scenario, for somewhat heavier stops, may be obtained in the presence of light singlets. An interesting bonus of this scenario is the possibility of explaining an excess of events observed in CP-even Higgs searches at LEP2.

Keywords: Beyond Standard Model, Higgs Physics, Supersymmetric Standard Model

ARXIV EPRINT: 1611.02353 


\section{Contents}

$\begin{array}{llr}1 & \text { Introduction } & 1\end{array}$

2 Enhanced tth coupling with a light singlet $\quad 3$

$3 \quad$ NMSSM realization $\quad 4$

$\begin{array}{llr}4 & \text { Higgs signal rates } & 8\end{array}$

5 Non-SM-like Higgs phenomenology 9

6 Conclusions 14

\section{Introduction}

After the discovery of a $125 \mathrm{GeV}$ resonance in July 2012 [1, 2], the LHC collaborations have established that its properties are close to the ones of the SM Higgs boson, namely a neutral CP-even scalar. The ratio of the observed Higgs production cross section to the SM predicted values differs from one by just a few tens of percent in most final state channels [3]. This indicates that the couplings to third generation fermions and vector gauge bosons are roughly consistent with their SM predicted values. The measurement of the Higgs production rate in association with top quarks in multilepton channels, however, shows central values that are significantly above the SM expectations [4-8]. Moreover, the search for Higgs bosons produced in association with weak gauge bosons and decaying into bottom quarks have revealed values that tend to be significantly smaller than the SM predicted values. In fact, if one ignores the correlation between the top-quark and gluon couplings to the Higgs, the best fit to the Higgs couplings leads to central values of the Higgs couplings to bottom-quarks and top-quarks that are $2 \sigma$ away from the SM predicted values, as shown by the recent analysis of the Higgs data by the CMS and ATLAS collaborations [3]. Although these channels are statistically limited, as reflected by the fact that no Higgs discovery may be established in the $t \bar{t} h$ channel at this point, it is worthwhile to analyze if such deviations from the SM predictions may be realized in any realistic low energy extension of the SM.

As has been shown in ref. [9], a Higgs with enhanced couplings to the top quark and suppressed couplings to the bottom quark may be easily obtained in two Higgs doublet models $(2 \mathrm{HDM})$, for values of $\tan \beta$, the ratio of vacuum expectation value, close to one. The trouble with these models is that the coupling of the Higgs to gluons is in first approximation proportional to the Higgs coupling to the top quark and therefore one would expect enhanced gluon fusion production rates too, what is in conflict with experiment. In addition, the bottom coupling suppression would lead to a reduction of the width of the 
Higgs decay into bottom quarks, and to a subsequent enhancement of the branching ratios of the Higgs decay into vector gauge bosons.

A consistent solution to this problem may be obtained in the presence of additional light color degrees of freedom, with significant couplings to the Higgs and that may lead to contributions to the gluon coupling that compensates the top-quark ones. ${ }^{1}$ A particular example is the case of low-energy supersymmetry [12], in which these new colored particles are just given by the stops, the superpartners of the top quark. It was demonstrated that if the lightest stop mass is of the order of a few hundred $\mathrm{GeV}$ and the stop mixing parameters are large, the Higgs rates may be brought to agreement with the experimentally observed ones [9]. This solution cannot be realized in the Minimal Supersymmetric extension of the SM (MSSM) since it is difficult to obtain the right Higgs mass for such small values of $\tan \beta$ and the stop masses and, in addition, the Higgs mixing effects in this model lead to an enhancement of the Higgs bottom coupling and a suppression of the top one, that is the opposite as the tendency indicated by data.

In ref. [9] it was shown that this problem may be fixed in the simplest extension of the MSSM, with the addition of a singlet superfield, namely the NMSSM [13]. In such a case, the same coupling $\lambda$ that enhances the Higgs mass modifies the CP-even Higgs mixing, leading to couplings of the Higgs consistent with the observed ones. It was shown that, for heavy singlets, the required values of $\lambda$, the superpotential coupling of the singlet to the Higgs doublet superfields, were $\lambda>0.7$, what leads to the breakdown of the perturbative consistency of the theory below the GUT scale. In this article, we shall show that an alternative solution can be obtained for the case of light singlets. In such a case, the values of $\lambda$ can be lower than 0.7 and a perturbative consistent solution can be found. In addition, although this is not a requirement of this scenario, we shall demonstrate that values of the masses and couplings to vector bosons of the (predominantly singlet) lightest CP-even Higgs, consistent with the ones required to explain an observed excess in the LEP2 Higgs search data [14], can be obtained in this scenario.

The possibility of a tth coupling enhancement was also studied in the context of models with vector-like top quark partners that mix with the top quark [15]. The $t \bar{t} h$ production excess is mainly driven by the multi-lepton channel and is one of several excesses in searches in final states consisting of bottom quarks and many leptons [16-18]. Such excesses were also explained in models with standard Higgs sector by introducing new scalar or fermion particles [19-21].

This article is organized as follow. In section 2 we review the conditions to obtain an enhancement of the Higgs-top-quark coupling in the presence of light singlets. In section 3 we present the NMSSM realization and in section 4 we present a numerical analysis of the Higgs production rates in this scenario. In section 5 we analyze the phenomenology of the non-standard Higgs bosons. We reserve section 6 for our conclusions.

\footnotetext{
${ }^{1}$ The degeneracy in the gluon fusion production cross-section between the top quark and New Physics contributions can be broken by studying production of a boosted Higgs with a jet, see e.g. [10, 11].
} 


\section{Enhanced $t$ th coupling with a light singlet}

In order to enhance the associated production of $h$ with a top quark pair (which we shall denote $t$ th) in a model with two Higgs doublets the $125 \mathrm{GeV}$ Higgs eigenstate must have a non-negligible component in the non-SM-like doublet. In type-II 2HDM the CP-even Higgs couplings to fermions and gauge bosons (normalized to the SM values) are determined by the values of the CP-even Higgs mixing angle $\alpha$ and $\tan \beta$, the ratio of the two Higgs doublet vacuum expectation values in the following way:

$$
\begin{aligned}
c_{t} & =\frac{\cos \alpha}{\sin \beta}=\sin (\beta-\alpha)[1+\cot \beta \cot (\beta-\alpha)], \\
c_{b} & =-\frac{\sin \alpha}{\cos \beta}=\sin (\beta-\alpha)[1-\tan \beta \cos (\beta-\alpha)], \\
c_{V} & =\sin (\beta-\alpha),
\end{aligned}
$$

where $c_{i}=g_{h i i} / g_{h i i}^{\mathrm{SM}}$ denotes the ratio of the Higgs coupling to the $i$ particle normalized to its $\mathrm{SM}$ value. Enhanced $t$ th is obtained for low $\tan \beta$ and sizeable $\cot (\beta-\alpha)>0$. In a pure $2 \mathrm{HDM}$, in which the coupling of the Higgs bosons to gluons is induced by loops of third generation quarks, enhanced tth is inevitably correlated with enhanced Higgs production in the gluon fusion channel, which is phenomenologically unacceptable. One can avoid this correlation if there exist additional light colored states, such as stops, that give negative contribution to the effective Higgs coupling to gluons [9]. It was demonstrated in ref. [9] that in such a case enhancement of the $t$ th signal of the Higgs decaying to gauge bosons can be larger than in the SM by a factor of two without violation of any experimental constraints. Moreover, it was shown in ref. [9] that this can be also realized in the NMSSM with heavy singlets, which effectively corresponds to a type-II $2 \mathrm{HDM}$, with relatively light highly-mixed stops. The stop contributions modify the effective Higgs coupling to gluons and photons in the following way, see e.g. refs. [22, 23]:

$$
\frac{c_{g}}{c_{g}^{\mathrm{SM}}}=\frac{c_{\gamma}}{c_{\gamma}^{\mathrm{SM}}}=c_{t}+\frac{m_{t}^{2}}{4}\left[c_{t}\left(\frac{1}{m_{\tilde{t}_{1}}^{2}}+\frac{1}{m_{\tilde{t}_{2}}^{2}}\right)-\frac{\tilde{X}_{t}^{2}}{m_{\tilde{t}_{1}}^{2} m_{\tilde{t}_{2}}^{2}}\right],
$$

where $\tilde{X}_{t}^{2} \equiv X_{t}\left(A_{t} \frac{\cos \alpha}{\sin \beta}+\mu \frac{\sin \alpha}{\sin \beta}\right)$ with the stop mixing parameter given by $X_{t} \equiv A_{t}-$ $\mu / \tan \beta$ (note: in the decoupling limit $\tilde{X}_{t}^{2}=X_{t}^{2}$ ). It should be clear from the above formula that significant reduction of the Higgs coupling to gluons is possible only for light enough stops. At the ICHEP 2016 conference, the LHC collaborations presented new constraints on the lightest stop mass, which are quite strong in simplified models in which the mass difference between the stop and the lightest supersymmetric particle is large [2428]. However, the constraints are still relatively weak for small mass splitting between the stop and the LSP. In order to avoid constraints on light sbottoms it is preferred that the lightest stop is mostly right-handed. The limit is especially weak if the lightest stop decays mainly to charm and neutralino. For such topology a lightest stop as light as about $260 \mathrm{GeV}$ is allowed if the stop-LSP mass splitting is above about $20 \mathrm{GeV}$ (but not large enough to make the stop decays to charm and neutralino subdominant) [29-32]. 
Another important requirement to induce a large tth enhancement in the NMSSM with heavy singlets is that the value of $\lambda \gtrsim 0.8$ [9]. Such large values of $\lambda$ lead to a Landau pole below the Grand Unification (GUT) scale, $M_{\mathrm{GUT}} \simeq 210^{16} \mathrm{GeV}$. In the following, we will show that smaller values of $\lambda$ are possible if the singlets are light.

If in addition to the CP-even Higgs bosons proceeding from the two Higgs doublets also a singlet is light, in order to determine non-SM doublet component of the $125 \mathrm{GeV}$ Higgs one needs to consider three-by-three Higgs mass matrix (in the Higgs basis): ${ }^{2}$

$$
\hat{M}^{2}=\left(\begin{array}{ccc}
\hat{M}_{h h}^{2} & \hat{M}_{h H}^{2} & \hat{M}_{h s}^{2} \\
\hat{M}_{h H}^{2} & \hat{M}_{H H}^{2} & \hat{M}_{H s}^{2} \\
\hat{M}_{h s}^{2} & \hat{M}_{H s}^{2} & \hat{M}_{s s}^{2}
\end{array}\right) .
$$

Since the singlet does not couple to SM particles, the couplings of the $125 \mathrm{GeV}$ are still given by eqs. (2.1)-(2.3) but the effective $\cot (\beta-\alpha)$ depends now also on the mixing of the singlet with the Higgs doublets:

$$
\cot (\beta-\alpha)=\frac{\left(m_{h}^{2}-\hat{M}_{h h}^{2}\right) \hat{M}_{H s}^{2}+\hat{M}_{h s}^{2} \hat{M}_{h H}^{2}}{\left(\hat{M}_{H H}^{2}-m_{h}^{2}\right) \hat{M}_{h s}^{2}-\hat{M}_{H s}^{2} \hat{M}_{h H}^{2}} .
$$

In the above the effective $\sin (\beta-\alpha)$ and $-\cos (\beta-\alpha)$ are defined as the $\hat{h}$ and $\hat{H}$ components of $h$, respectively. We see that it is possible to generate non-zero $\cot (\beta-\alpha)$ even for $\hat{M}_{h H}^{2}=0$. This feature is particularly important in the context of the NMSSM since for the most interesting values of $\lambda \approx 0.65$ (which give a large positive correction to the Higgs mass but do not generate the Landau pole below the GUT scale) $\hat{M}_{h H}^{2} \approx 0$ which is known as the NMSSM alignment limit $[33,34]$. Notice also that in the limit $\hat{M}_{h H}^{2}=0$, tth is enhanced for $\hat{M}_{h s}^{2} \hat{M}_{H s}^{2}>0(<0)$ if the singlet-like scalar is lighter (heavier) than $125 \mathrm{GeV}$. This is because in this limit mixing of the Higgs with lighter (heavier) singlet-like scalar pushes up (down) the Higgs mass so $\left(m_{h}^{2}-\hat{M}_{h h}^{2}\right)$ is positive (negative) [35-37]. It is also interesting to note that in principle large $\cot (\beta-\alpha)$ may be also obtained for very heavy non-SM Higgs doublet if $\left|\hat{M}_{H s}^{2}\right|$ is large enough.

\section{NMSSM realization}

Let us now focus on $t$ th enhancement in the general NMSSM for which the MSSM superpotential is supplemented by:

$$
W_{\mathrm{NMSSM}}=\lambda S H_{u} H_{d}+\xi_{F} S+\mu^{\prime} S^{2} / 2+\kappa S^{3} / 3 .
$$

\footnotetext{
${ }^{2}$ The Higgs basis $(\hat{h}, \hat{H}, \hat{s})$ is defined as $\hat{h}=H_{d} \cos \beta+H_{u} \sin \beta, \hat{H}=H_{d} \sin \beta-H_{u} \cos \beta$ and $\hat{s}=S$. In this basis the $\hat{h}$ field has exactly the same couplings to the gauge bosons and fermions as the SM Higgs field. The field $\hat{H}$ is a non-SM-like doublet which does not couple to the gauge bosons and its couplings to the down and up fermions are the SM Higgs ones rescaled by $\tan \beta$ and $-\cot \beta$, respectively. The mass eigenstates are denoted as $s, h, H$, with the understanding that $h$ is the SM-like Higgs.
} 
and the soft terms are given by

$$
\begin{aligned}
-\mathcal{L}_{\text {soft }} \supset & m_{H_{u}}^{2}\left|H_{u}\right|^{2}+m_{H_{d}}^{2}\left|H_{d}\right|^{2}+m_{S}^{2}|S|^{2} \\
& +\left(A_{\lambda} \lambda H_{u} H_{d} S+\frac{1}{3} A_{\kappa} \kappa S^{3}+m_{3}^{2} H_{u} H_{d}+\frac{1}{2} m_{S}^{\prime 2} S^{2}+\xi_{S} S+\text { h.c. }\right),
\end{aligned}
$$

where $S$ is a SM-singlet superfield which scalar component acquires vacuum expectation value $v_{s}$. The first term is the source of the effective higgsino mass parameter, $\mu_{\text {eff }} \equiv \lambda v_{s}$ (we drop the subscript "eff" in the rest of the paper and we set explicitly the MSSM $\mu$-term to zero by shifting the value of $S$, what amounts to a simple redefinition of some of the parameters).

The off-diagonal terms of the tree-level Higgs mass matrix in the Higgs basis in the general NMSSM are given by:

$$
\begin{aligned}
\hat{M}_{h H}^{2} & =\frac{1}{2}\left(M_{Z}^{2}-\lambda^{2} v^{2}\right) \sin 4 \beta, \\
\hat{M}_{h s}^{2} & =\lambda v(2 \mu-\Lambda \sin 2 \beta), \\
\hat{M}_{H s}^{2} & =\lambda v \Lambda \cos 2 \beta .
\end{aligned}
$$

where $\Lambda \equiv A_{\lambda}+\mu^{\prime}+2 \kappa v_{s}, v \approx 174 \mathrm{GeV}$, and we have ignored loop-corrections that are generically small and have a minor phenomenological impact in the region of parameters relevant for this work $[33,34]$. The diagonal mass in the SM-like Higgs component is given by:

$$
\hat{M}_{h h}^{2}=M_{Z}^{2} \cos ^{2}(2 \beta)+\lambda^{2} v^{2} \sin ^{2}(2 \beta)+\Delta_{\text {loop }},
$$

where $\Delta_{\text {loop }}$ parameterizes loop corrections which are dominated by stops.

The explicit form of the remaining diagonal entries of the Higgs mass matrix, that can be found e.g. in ref. [37], is not relevant for our discussion, since in the general NMSSM there is enough freedom in the parameter space to set them to arbitrary values.

Eq. (3.6) implies that for small values of $\tan \beta$ the Higgs mass of $125 \mathrm{GeV}$ can be accommodated only for relatively large $\lambda \gtrsim 0.5$ unless stops are heavy. On the other hand, avoiding a Landau pole below the GUT scale sets requires $\lambda \lesssim 0.7$ (for $\kappa \ll \lambda$ ) with the upper bound getting stronger as $\tan \beta$ decreases. For this range of $\lambda$ the lack of a Landau pole below the GUT scale for the top Yukawa coupling requires also $\tan \beta \gtrsim 1.5$. As a result, in this range of parameters $\hat{M}_{h H}^{2} \approx 0$ so using eqs. (3.4)-(3.5) together with eq. (2.6) one obtains the following approximate formula for the effective $\cot (\beta-\alpha)$ :

$$
\cot (\beta-\alpha) \approx \frac{\left(m_{h}^{2}-\hat{M}_{h h}^{2}\right) \lambda v \Lambda \cos 2 \beta}{\left(\hat{M}_{H H}^{2}-m_{h}^{2}\right) \hat{M}_{h s}^{2}} \approx \operatorname{sgn}\left(\frac{\Lambda \sin 2 \beta-2 \mu}{m_{h}-m_{s}}\right) \bar{g}_{s} \frac{\lambda v \Lambda \cos 2 \beta}{\hat{M}_{H H}^{2}-m_{h}^{2}},
$$

where $\bar{g}_{s}$ is the $s$ coupling to the $Z$ boson normalized to the corresponding coupling of the SM Higgs with the same mass. In the approximation made on the very right hand side of the above equation we assumed $\bar{g}_{s}^{2} \ll 1$ which is justified since the Higgs-singlet mixing must be relatively small to comply with constraints from the LHC and LEP. Smallness of the Higgs-singlet mixing requires $\left|\hat{M}_{h s}^{2}\right| \ll m_{h}^{2}+m_{s}^{2}$ which is fulfilled when

$$
\Lambda \approx \frac{2 \mu}{\sin 2 \beta},
$$


and implies in particular that $\mu \Lambda$ must be positive. We should stress, however, that the above condition should not be satisfied exactly because otherwise $\cot (\beta-\alpha)$ would vanish. Using the above condition together with eq. (3.7) one obtains an approximate formula for the effective $\cot (\beta-\alpha)$ as a function of $\mu$, namely

$$
\cot (\beta-\alpha) \approx \operatorname{sgn}\left(\frac{\Lambda \sin 2 \beta-2 \mu}{m_{h}-m_{s}}\right) \bar{g}_{s} \frac{\lambda v \mu \cot 2 \beta}{\hat{M}_{H H}^{2}-m_{h}^{2}} .
$$

In order to enhance $t$ th signal one has to also guarantee that $\cot (\beta-\alpha)>0$ which happens if the following condition is fulfilled:

$$
|\Lambda|>\frac{2|\mu|}{\sin 2 \beta} \quad\left(|\Lambda|<\frac{2|\mu|}{\sin 2 \beta}\right)
$$

for $m_{s}<m_{h}\left(m_{s}>m_{h}\right)$.

Eq. (3.9) confirms the intuitive expectation that large $t$ th enhancement prefers relatively light MSSM-like Higgs bosons. However, it also shows that large $|\mu|$ is preferred and that $H$ does not have to be very light if $|\mu|$ is large enough. The importance of $|\mu|$ can be seen from table 1 where several benchmark points with large tth enhancement are presented. We shall define the theoretically predicted signal strengths modifiers as:

$$
R_{i}^{j} \equiv \frac{\sigma^{j} \times \mathrm{BR}(h \rightarrow i)}{\sigma^{j \mathrm{SM}} \times \mathrm{BR}^{\mathrm{SM}}(h \rightarrow i)}, .
$$

and we shall distinguish the theoretical predictions for the signal strengths from the corresponding LHC measurements, that we define in the conventional way as $\mu_{i}^{j}$. Comparing points $\mathrm{P} 3$ and $\mathrm{P} 4$ we see that similar $t$ th enhancement is possible for $M_{A}=300 \mathrm{GeV}$ and $\mu=500 \mathrm{GeV}$ as for $M_{A}=400 \mathrm{GeV}$ and $\mu=650 \mathrm{GeV}$ with comparable Higgs-singlet mixing. Eq. (3.9) also implies that the effective $|\cot (\beta-\alpha)|$ increases with $\tan \beta$. This is the reason why points $\mathrm{P} 1$ and $\mathrm{P} 3$ have similar values of $R_{V V}^{\mathrm{tth}}$ in spite of the fact that $\mathrm{P} 3$ features smaller Higgs-singlet mixing and the value of $\mu$ while the value of $M_{A}$ is the same.

Another interesting feature of this scenario is that light singlet-like scalar can explain the LEP2 excess [14]. Indeed, the LEP2 experiments observed an excess of $b \bar{b}$ events, produced in association with a $Z$ gauge boson, with an invariant mass in the vicinity of $98 \mathrm{GeV}$ and a signal strength of about one tenth of the one of the SM Higgs with the same mass. Phenomenological aspects of the NMSSM with singlet-like scalar explaining the LEP2 excess were studied e.g. in refs. [41-43] while in refs. [44, 45] it was shown that this excess can be explained in a UV complete NMSSM model based on gauge mediated SUSY breaking [46]. However, none of those works links the LEP2 excess to the $t$ th enhancement. In table 1 we give a value for the prediction of this signal strength:

$$
\xi_{b \bar{b}}^{\mathrm{LEP}} \equiv \bar{g}_{s}^{2} \times \frac{\mathrm{BR}(s \rightarrow b \bar{b})}{\mathrm{BR}\left(h^{\mathrm{SM}} \rightarrow b \bar{b}\right)},
$$

Note that the SM normalized $s b \bar{b}$ coupling in the present scenario is enhanced with respect to the corresponding $s Z Z$ coupling so $\xi_{b \bar{b}}^{\mathrm{LEP}}>\bar{g}_{s}^{2}$. Wee see that point $\mathrm{P} 1$ fits very well the LEP2 excess since it features $m_{s} \approx 98 \mathrm{GeV}$ and $\xi_{b \bar{b}}^{\mathrm{LEP}} \approx 0.1$. Point P2 also has $m_{s} \approx$ 


\begin{tabular}{|c|cccc|}
\hline & $\mathrm{P} 1$ & $\mathrm{P} 2$ & $\mathrm{P} 3$ & $\mathrm{P} 4$ \\
\hline$\lambda$ & 0.5 & 0.52 & 0.5 & 0.52 \\
$\tan \beta$ & 1.6 & 1.6 & 2 & 2 \\
\hline$m_{Q_{3}}$ & 800 & 800 & 800 & 800 \\
$m_{U_{3}}$ & 240 & 250 & 235 & 200 \\
$A_{t}$ & -1500 & -1500 & -1550 & -1480 \\
\hline$\mu$ & 550 & 650 & 500 & 650 \\
$\mu^{\prime}$ & 310 & 350 & 235 & 250 \\
$M_{A}$ & 300 & 300 & 300 & 400 \\
$M_{P}$ & 244 & 308 & 297 & 305 \\
$A_{\lambda}$ & 810 & 963 & 908 & 1305 \\
\hline$m_{s}$ & 98 & 98 & 88 & 84 \\
$m_{h}$ & 124.9 & 125.6 & 124.9 & 126.3 \\
$m_{H}$ & 303 & 336 & 355 & 414 \\
$m_{H^{ \pm}}$ & 217 & 199 & 224 & 317 \\
$m_{a}$ & 108 & 94 & 105 & 87 \\
$m_{A}$ & 311 & 358 & 359 & 434 \\
$m_{\tilde{\chi}_{1}^{0}}$ & 235 & 235 & 235 & 235 \\
$m_{\tilde{t}_{1}}$ & 272 & 277 & 276 & 275 \\
$m_{\tilde{t}_{2}}$ & 946 & 951 & 945 & 948 \\
\hline$R_{V V}^{\mathrm{th}}$ & 1.60 & 1.62 & 1.60 & 1.60 \\
$R_{\gamma \gamma}^{\mathrm{th}}$ & 1.83 & 1.85 & 1.80 & 1.78 \\
$R_{V V}^{\mathrm{gg}}$ & 1.02 & 1.00 & 1.04 & 1.04 \\
$R_{\gamma \gamma}^{\mathrm{gg}}$ & 1.16 & 1.15 & 1.16 & 1.16 \\
$R_{V V}^{\mathrm{VBF} / \mathrm{VH}}$ & 1.32 & 1.34 & 1.41 & 1.39 \\
$R_{\gamma \gamma}^{\mathrm{VBF} / \mathrm{VH}}$ & 1.51 & 1.54 & 1.58 & 1.55 \\
$R_{\tau \tau}^{\mathrm{VBF} / \mathrm{VH}}$ & 0.72 & 0.76 & 0.77 & 0.73 \\
\hline$\xi_{b \bar{b}}^{\mathrm{LEP}}$ & 0.10 & 0.06 & 0.04 & 0.06 \\
$\bar{g}_{s}$ & 0.30 & 0.24 & 0.20 & 0.24 \\
\hline
\end{tabular}

Table 1. List of benchmark points obtained with NMSSMTools 5.0.0 [38, 39]. All masses are in $\mathrm{GeV}$. All points satisfy all experimental constraints from the Higgs signal strength measurements, as well as from direct searches for Higgs bosons, checked with HiggsBounds 4.3.1 [40], and stops. The gluino and the remaining soft sfermion masses are set to $2 \mathrm{TeV}, M_{2}=1 \mathrm{TeV}, M_{1}=-235 \mathrm{GeV}$. All the remaining $A$-terms are set to $1.5 \mathrm{TeV}$, while $\kappa=A_{\kappa}=m_{3}^{2}=m_{S}^{\prime 2}=0$. The remaining parameters are calculated with NMSSMTools using EWSB conditions and the values of $\mu, M_{A}$ (diagonal mass of MSSM-like pseudoscalar) and $M_{P}$ (diagonal mass of singlet-like pseudoscalar). 
$98 \mathrm{GeV}$ but smaller Higgs-singlet mixing, hence also $\xi_{b \bar{b}}^{\mathrm{LEP}}$, than P1 so in order to have $t$ th enhancement of similar size $|\mu|$ is larger in P2 than in P1.

Even though it is an interesting possibility that this scenario can simultaneously explain the tth enhancement and the LEP2 excess, we should emphasize that our scenario does not require to have the singlet-like scalar mass to be close to the one necessary to explain the LEP excess. It is the size of the Higgs-singlet mixing rather than $m_{s}$ which controls the magnitude of the $t$ th enhancement as can be seen from benchmarks P3 and P4 that feature $m_{s}$ far away from the one consistent with the LEP excess. It is noteworthy that $m_{s}$ can be as small as about $85 \mathrm{GeV}$ (or even smaller if $|\mu|$ is larger than in benchmark P4) without inducing a conflict with stringent LEP constraints.

\section{Higgs signal rates}

All benchmark points presented in table 1 are compatible with the combination of the run-I Higgs signal measurements [3] at least at the $2 \sigma$ level. Nevertheless, there are some potential tensions of this scenario with recent run II data that will be probed with future LHC measurements. Let us now discuss these deviations and how they depend on the model parameters in some more detail. Let us start with the $\gamma \gamma$ decay channel in the gluon fusion production mode. All benchmarks were chosen to have $R_{\gamma \gamma}^{\mathrm{gg}} \approx 1.15$ by appropriate adjustment of the stop sector parameters. This is very close to the central value of the ATLAS and CMS combination of the Run-I data which yields $\mu_{\gamma \gamma}^{\mathrm{gg}}=1.10_{-0.22}^{+0.23}[3]$. Preliminary results of Run II indicate that both experiments observed some suppression of this signal strength: $\mu_{\gamma \gamma}^{\mathrm{gg}}=0.59_{-0.28}^{+0.29}$ for ATLAS [47] and $\mu_{\gamma \gamma}^{\mathrm{gg}}=0.77_{-0.23}^{+0.25}$ for CMS [48] so the $2 \sigma$ upper bound from ATLAS Run II on $R_{\gamma \gamma}^{\mathrm{gg}}$ is about 1.17. By the end of this year the LHC is expected to deliver a few times more data than analysed for ICHEP2016 so the discrepancy between the Run-I and Run-II results should be clarified relatively soon.

While the mechanism that we propose may generate some tension with the Run-II measurement of $\mu_{\gamma \gamma}^{\mathrm{gg}}$ the fit to the Run-II results for $h \rightarrow \gamma \gamma$ in the VBF production mode is significantly improved. Indeed, the ATLAS Run-II result is $\mu_{\gamma \gamma}^{\mathrm{VBF}}=2.24_{-0.71}^{+0.80}$ which is almost $2 \sigma$ above the SM prediction. It can be seen from the benchmark table that in the present scenario it is generically brought to $1 \sigma$ agreement with the ATLAS result. Moreover, the prediction is very close to the central value of the Run-II CMS result of $\mu_{\gamma \gamma}^{\mathrm{VBF}}=1.61_{-0.8}^{+0.9}$.

As already emphasized, the present scenario features also suppressed Higgs coupling to down-type fermions. This is in very good agreement with very weak signal of the Higgs decaying into $b \bar{b}$ observed in both Run I and Run II of the LHC. On the other hand, the Higgs decays to $\tau \tau$ in the VBF production mode (gluon fusion is far less sensitive in this decay channel) has been observed to be relatively close to the SM prediction. Nevertheless, due to the large uncertainties, values of $R_{\tau \tau}^{\mathrm{VBF} / \mathrm{VH}}$ as small as about $0.4(0.8)$ are consistent with current data at $2 \sigma(1 \sigma)$ level [3]. We can see from table 1 that $R_{\tau \tau}^{\mathrm{VBF} / \mathrm{VH}}$ is in the range between 0.7 and 0.8 for $R_{V V}^{\mathrm{tth}}$ of about 1.6. Larger values of $R_{\tau \tau}^{\mathrm{VBF} / \mathrm{VH}}$ keeping the same value of $R_{V V}^{\mathrm{tth}}$ are obtained for smaller Higgs-singlet mixing which is evident from comparison of benchmark P1 with the other ones. Needless to say that $R_{\tau \tau}^{\mathrm{VBF} / \mathrm{VH}}$ also deviates less from the SM prediction when $R_{V V}^{\mathrm{tth}}$ is smaller. It is also interesting to note 
that a given value of $R_{V V}^{\mathrm{tth}}$ fitting the LEP2 excess fixes $R_{\tau \tau}^{\mathrm{VBF} / \mathrm{VH}}$ (up to small variations from $\tan \beta$ dependence), e.g. $R_{V V}^{\mathrm{tth}} \approx 1.6$ implies $R_{\tau \tau}^{\mathrm{VBF} / \mathrm{VH}} \approx 0.7$ as for benchmark P1. Such indirect cross-check is particularly important since in this scenario the CP-even singlet $s$ decays are predominantly into $b \bar{b}$ final states.

Let us also note that the larger $\tan \beta$ is the more enhancement of $R_{V V}^{\mathrm{tth}}$ comes from suppressed $\mathrm{BR}(h \rightarrow b \bar{b})$ which results in closer values for signal rates in tth and $\mathrm{VBF} / \mathrm{VH}$ production modes. Therefore, if the excess in the $t$ th channel persists, information about $\tan \beta$ may be also extracted from future Higgs precision measurements.

We should also comment that all benchmarks presented in table 1 feature rather large values of $\left|X_{t}\right|$ with $\left|X_{t}\right| / m_{\tilde{t}_{2}}$ around two. Such values are needed to keep the gluon fusion signal rates close to SM predictions when the $t$ th signals are enhanced. It is known from MSSM studies that too large values of $\left|A_{t}\right|$ may lead to color and/or charge breaking global minima [49]. Indeed, all the benchmarks in table 1 feature unphysical global minima. Whether these benchmarks are phenomenologically viable depends on the lifetime of the metastable EW vacuum. It was recently shown that constraints on $\left|A_{t}\right|$ from stability of the EW vacuum are overestimated by analytical formulae, presented e.g. in ref. [49], if one admits as a viable solution sufficiently long-lived metastable EW vacua [50-52]. It was also emphasized in ref. [52] that it is essentially impossible to have a simple universal phenomenological formula that may reliable estimate whether a given point in the MSSM parameter space leads to destabilization of the $\mathrm{EW}$ vacuum and a dedicated study is necessary. Moreover, the MSSM analyses considered only moderate and large $\tan \beta$, which is not our case. Last but not least, the NMSSM scalar potential has a richer structure than the MSSM one. Studies of color and charge breaking vacua in the scale-invariant NMSSM have been performed in the past but they either consider NMSSM specific directions [53, 54] in field space or give a simple generalizations of the MSSM analytical formulae [55] which do not account for the fact that EW vacuum may be metastable and long-lived. It is beyond the scope of the present paper to investigate the conditions for metastability of the EW vacuum in the general NMSSM and we leave a dedicated study of this issue for future work. Nevertheless, we expect that for a given value of the lightest stop mass there exist an upper bound on $\left|X_{t}\right| / m_{\tilde{t}_{2}}$ which could be translated to an upper bound on possible $t$ th enhancement after taking into account the Higgs measurements in the gluon fusion production modes.

\section{Non-SM-like Higgs phenomenology}

The phenomenology of non-SM-like Higgs bosons is significantly different from that in the case of heavy singlet discussed in ref. [9]. Both, light and heavy singlet scenarios feature heavy doublet-like Higgs bosons in the range of several hundred $\mathrm{GeV}$ but their dominant decay channels are totally different. In the heavy singlet case $H$ and $A$ have large branching ratio for decay into a pair of lightest neutralinos and $H \rightarrow h h$ decay is also very frequent if kinematically accessible. In contrast, in the light singlet scenario $H / A$ decay products in dominant channels involve light singlet-like (pseudo)scalars, as can be seen from table 3 . The main decay mode for $A$ is as and $Z s$. $\operatorname{BR}\left(A \rightarrow H^{ \pm} W^{\mp}\right)$ may also be large if this decay channel is kinematically open. The main decay channels for $H$ involve $a a, a Z$ and 
sometimes $s s$ and $H^{ \pm} W^{\mp}$. The only existing analysis that may probe the sector of heavy neutral Higgses is the CMS search for $A \rightarrow Z s$ or $H \rightarrow Z a$ with $Z$ decaying leptonically and the lighter pseudo(scalar) decaying to $b \bar{b}$. Branching ratios of $A \rightarrow Z s$ and $H \rightarrow Z a$ vary between 10 and $40 \%[58,59]$. The upper limits on the $13 \mathrm{TeV}$ cross-section for $l l b \bar{b}$ production in this topology are between 0.1 and $1 \mathrm{pb}$ (depending on the masses of the heavy and light Higgs bosons) with $2.3 \mathrm{fb}^{-1}$ of $13 \mathrm{TeV}$ CMS data. This is at least factor of few above the prediction for this cross-section for the benchmark points presented in table 3 . Therefore, with $\mathcal{O}(50) \mathrm{fb}^{-1}$ it may be possible to probe some of the parameter space that predicts $t$ th enhancement but for a generic point in parameter space this would require much more data. Searches for double Higgs production in $b \bar{b} b \bar{b}$ final state may be more powerful to test this model provided that the experimental collaborations will relax the assumption that the products of heavy resonance decay have mass of $125 \mathrm{GeV}$ as it is assumed in existing analyses [60,61]. It is also noteworthy that $H \rightarrow h h$ decays are negligible in the light singlet scenario which is due to the fact that the correct Higgs mass requires $\lambda \approx 0.5$ for which $H h h$ coupling is strongly suppressed due to approximate alignment in the $\hat{h}-\hat{H}$ sector [33, 34].

The charged Higgs is also relatively light. It is typically not far above $H^{ \pm} \rightarrow t b$ threshold while the ATLAS Run-II results for charged Higgs search in $t \bar{b}$ decay mode are available only for $m_{H^{ \pm}}>300 \mathrm{GeV}$ with the strongest upper bound on $\tan \beta$ in type-II $2 \mathrm{HDM}$ with $\mathrm{BR}\left(H^{ \pm} \rightarrow t b\right)=100 \%$ of about 1.7 for $m_{H^{ \pm}}=300 \mathrm{GeV}$ [62]. While the charged Higgs production cross-section in our model is expected to be similar as in the type-II $2 \mathrm{HDM}, \mathrm{BR}\left(H^{ \pm} \rightarrow t b\right)$ is much less than $100 \%$ since charged Higgs often decays to $W^{+} a$ and $W^{+} s$ which weakens the bound on $\tan \beta$ significantly. This is the reason why benchmark P4 which features $m_{H^{ \pm}}>300 \mathrm{GeV}$ comfortably satisfies current constraints. Nevertheless, future searches for charged Higgs in $t \bar{b}$ decay mode may probe important part of the model parameter space, especially if they are extended to masses below $300 \mathrm{GeV}$. While there are difficulties in reliable computation of the charged Higgs production crosssection for masses close to the top quark mass, some progress has been made on this front recently [63], so comparing the experimental results with theoretical predictions may be easier now. Moreover, it was argued in ref. [64] that the NMSSM charged Higgs decaying to $W^{+} a$ could be efficiently probed in searches for events with missing transverse momentum, b-jets, leptons and/or taus.

As already emphasized, the crucial feature that allows for significant tth enhancement is large mixing between singlet scalar and MSSM-like doublet scalar. This generically implies also large mixing in the pseudoscalar sector. Indeed, for benchmarks presented in tables 13 this mixing is close to maximal. One consequence of this is large gluon-fusion production cross-section for lighter pseudoscalar, as seen from table 3. Direct production of $a$ may be discovered at the LHC in the $\tau \tau$ decay channel which contributes about $10 \%$ to total decay width of $a$ (the remaining $90 \%$ of $a$ decays are to $b \bar{b}$ but this decay channel is extremely difficult to observe in the gluon-fusion production mode at the LHC). On the other hand, CMS $\tau \tau$ search extends down to masses of about $90 \mathrm{GeV}$, so it covers an interesting part of parameter space of the model including benchmarks P1-P3. The current upper limit on $\sigma(g g a) \times \mathrm{BR}(a \rightarrow \tau \tau)$ from the $13 \mathrm{TeV}$ CMS search with $2.3 \mathrm{fb}^{-1}$ of data [65] is about 40 $\mathrm{pb}$ for the mass close to $100 \mathrm{GeV}$ with the limit improving rather quickly for masses above 


\begin{tabular}{|c|c|c|}
\hline & P5 & P6 \\
\hline$\lambda$ & 0.48 & 0.5 \\
\hline $\tan \beta$ & 1.6 & 2 \\
\hline$\kappa$ & 0.48 & 0.48 \\
\hline$m_{Q_{3}}$ & 800 & 800 \\
\hline$m_{U_{3}}$ & 195 & 190 \\
\hline$A_{t}$ & -1400 & -1450 \\
\hline$\mu$ & 700 & 700 \\
\hline$A_{\lambda}$ & -15 & 223 \\
\hline$A_{\kappa}$ & -3678 & -3431 \\
\hline$\xi_{S}$ & $-10^{9}$ & $-10^{9}$ \\
\hline$m_{3}^{2}$ & $-4.9 \cdot 10^{5}$ & $-6.1 \cdot 10^{5}$ \\
\hline$m_{S}^{\prime 2}$ & 0 & $-3.64 \cdot 10^{6}$ \\
\hline$m_{s}$ & 98 & 90 \\
\hline$m_{h}$ & 125.1 & 125.5 \\
\hline$m_{H}$ & 325 & 405 \\
\hline$m_{H^{ \pm}}$ & 246 & 271 \\
\hline$m_{a}$ & 2834 & 107 \\
\hline$m_{A}$ & 248 & 442 \\
\hline$m_{\tilde{\chi}_{1}^{0}}$ & 235 & 234 \\
\hline$m_{\tilde{t}_{1}}$ & 271 & 277 \\
\hline$m_{\tilde{t}_{2}}$ & 950 & 949 \\
\hline$R_{V V}^{\mathrm{tth}}$ & 1.59 & 1.59 \\
\hline$R_{\gamma \gamma}^{\mathrm{tth}}$ & 1.81 & 1.76 \\
\hline$R_{V V}^{\mathrm{gg}}$ & 1.03 & 1.05 \\
\hline$R_{\gamma \gamma}^{\mathrm{gg}}$ & 1.17 & 1.16 \\
\hline$R_{V V}^{\mathrm{VBF} / \mathrm{VH}}$ & 1.31 & 1.40 \\
\hline$R_{\gamma \gamma}^{\mathrm{VBF} / \mathrm{VH}}$ & 1.49 & 1.56 \\
\hline$R_{\tau \tau}^{\mathrm{VBF} / \mathrm{VH}}$ & 0.71 & 0.77 \\
\hline$\xi_{b \bar{b}}^{\mathrm{LEP}}$ & 0.11 & 0.04 \\
\hline $\bar{g}_{s}$ & 0.32 & 0.20 \\
\hline
\end{tabular}

Table 2. List of benchmark points that avoid unphysical global minimum. Unspecified MSSM-like parameters are the same as in table 1 while $\mu^{\prime}=\xi_{F}=0$. All dimensionful parameters are in $\mathrm{GeV}$ except for $\xi_{S}$ in $\mathrm{GeV}^{3}$ and $m_{3}^{2}$ and $m_{S}^{\prime 2}$ in $\mathrm{GeV}^{2}$. 


\begin{tabular}{|c|c|c|c|c|c|c|}
\hline & P1 & P2 & P3 & P4 & P5 & P6 \\
\hline $\mathrm{BR}(H \rightarrow t \bar{t})$ & 0 & 0 & 0.01 & 0.07 & 0 & 0.17 \\
\hline $\mathrm{BR}(H \rightarrow s s)$ & 0.32 & ¡0.01 & 0.05 & 0.28 & 0.72 & 0.01 \\
\hline $\mathrm{BR}(H \rightarrow a a)$ & 0.31 & 0.66 & 0.39 & 0.12 & 0 & 0.81 \\
\hline $\mathrm{BR}(H \rightarrow a Z)$ & 0.22 & 0.19 & 0.35 & 0.38 & 0 & 0.12 \\
\hline $\mathrm{BR}(H \rightarrow h s)$ & 0.11 & ¡0.01 & ¡0.01 & 0.11 & 0.24 & ¡0.01 \\
\hline $\operatorname{BR}\left(H \rightarrow H^{ \pm} W^{\mp}\right)$ & 0.01 & 0.13 & 0.19 & 0.03 & 0 & 0.05 \\
\hline $\mathrm{BR}(A \rightarrow t \bar{t})$ & 0 & 0.14 & 0.13 & 0.18 & 0 & 0.06 \\
\hline $\mathrm{BR}(A \rightarrow a s)$ & 0.66 & 0.39 & 0.32 & 0.35 & 0 & 0.61 \\
\hline $\mathrm{BR}(A \rightarrow Z s)$ & 0.22 & 0.23 & 0.33 & 0.32 & 0.95 & 0.14 \\
\hline $\mathrm{BR}(A \rightarrow a h)$ & 0.08 & ¡0.01 & ¡0.01 & 0.05 & 0 & 0.07 \\
\hline $\operatorname{BR}\left(A \rightarrow H^{ \pm} W^{\mp}\right)$ & 0.04 & 0.24 & 0.20 & 0.08 & 0 & 0.12 \\
\hline $\mathrm{BR}\left(H^{+} \rightarrow t \bar{b}\right)$ & 0.58 & 0.43 & 0.34 & 0.29 & 0.70 & 0.30 \\
\hline $\mathrm{BR}\left(H^{+} \rightarrow W^{+} a\right)$ & 0.19 & 0.34 & 0.27 & 0.38 & 0 & 0.34 \\
\hline $\mathrm{BR}\left(H^{+} \rightarrow W^{+} s\right)$ & 0.23 & 0.23 & 0.39 & 0.32 & 0.29 & 0.36 \\
\hline$\sigma(g g H)[\mathrm{pb}]$ & 2.4 & 1.6 & 1.2 & 1.4 & 2.7 & 1.2 \\
\hline$\sigma(g g A)[\mathrm{pb}]$ & 4.9 & 5.2 & 4.0 & 2.2 & 11.9 & 1.3 \\
\hline$\sigma(g g a)[\mathrm{pb}]$ & 24.3 & 40.4 & 18.7 & 19.2 & $10^{-9}$ & 17.7 \\
\hline$\sigma(g g s)[\mathrm{pb}]$ & 4.6 & 10.6 & 10.3 & 7.1 & 3.9 & 9.8 \\
\hline$\Omega_{\mathrm{th}} h^{2}$ & 0.08 & 0.09 & 0.07 & 0.06 & 0.08 & 0.07 \\
\hline$\sigma_{\mathrm{SI}}^{\mathrm{p}}[\mathrm{pb}]$ & $3.8 \cdot 10^{-10}$ & $3 \cdot 10^{-10}$ & $8.4 \cdot 10^{-10}$ & $4.3 \cdot 10^{-10}$ & $2.5 \cdot 10^{-10}$ & $3.8 \cdot 10^{-10}$ \\
\hline
\end{tabular}

Table 3. Branching ratios and gluon-fusion production cross-sections (calculated with SuShi 1.6.0 $[56,57])$ for non-SM-like Higgs bosons for benchmark points presented in tables 1 and 2. Thermal relic abundance $\Omega_{\mathrm{th}} h^{2}$ of the LSP and SI LSP-proton scattering cross-section $\sigma_{\mathrm{SI}}^{\mathrm{p}}$ are also given.

about $120 \mathrm{GeV}$. Therefore, the limit is an order of magnitude above the benchmark predictions so while some of the parameter space may be probed with $300 \mathrm{fb}^{-1}$, as exemplified by $\mathrm{P} 2$, vast of the parameter space will require high-luminosity LHC to be covered.

Weaker constraints for a ditau resonance with mass about $100 \mathrm{GeV}$ are also the reason why it is hard to probe the production of the $98 \mathrm{GeV}$ singlet scalar explaining the LEP excess. For benchmark P1 which fits the LEP excess the cross-section for $\tau \tau$ from decay of $s$ produced in gluon fusion is about 100 times smaller than the CMS upper limit so it might not be probed even at high-luminosity LHC. It is interesting to note that the benchmark P2 has larger gluon-fusion production cross-section of $s$ than P1 by more than a factor of two even though $s$ has much smaller $\hat{h}$ component than in P1. This is mainly because $\hat{h}$ and $\hat{H}$ components of $s$ give opposite sign contributions to $s$ coupling to top quarks, which is the main source of effective coupling to gluons. It turns out that these contributions have similar magnitude for values of $\bar{g}_{s}$ that can explain the LEP excess which results in a small $s$ coupling to top quarks, and hence also to gluons. For smaller values of $\bar{g}_{s}$ this cancellation is less efficient and a larger $\hat{H}-\hat{s}$ mixing is needed to keep the same th enhancement. In consequence, larger cross-sections for $s$ production via gluon fusion are predicted. 
Since scalars, unlike pseudoscalars, couple to weak gauge bosons at tree-level, $s$ can be also produced in VBF or VH modes. These modes have smaller production cross sections but also suffer from less background and for $125 \mathrm{GeV}$ scalars they turn out to be much more sensitive to the gluon-fusion mode both in $b \bar{b}$ and $\tau \tau$ channels. Unfortunately, the LHC collaborations have not looked at these production modes for masses below $100 \mathrm{GeV}$ so currently they cannot test the LEP2 excess. We strongly encourage them to extend their analyses to lower masses.

Let us also comment on the fact that in this scenario the LSP may be a good dark matter candidate. In the presented benchmarks the LSP is mainly a Bino, but its thermal relic abundance $\Omega_{\mathrm{th}} h^{2}$ (computed with microOMEGAs [66]) is in agreement with the upper bound on $\Omega h^{2}$ from Planck measurements [67]. This is because small mass splitting between the LSP and the lightest stop required to satisfy the LHC constraints results in efficient stop co-annihilations. Non-negligible LSP annihilation into final states involving light (pseudo)scalars is another reason for $\Omega_{\mathrm{th}} h^{2}$ much smaller than generically predicted for Bino-dominated LSP. In fact, for the benchmarks $\Omega_{\mathrm{th}} h^{2}$ ranges between 0.06 to 0.1 so is somewhat below the central value of 0.12 measured by Planck [67]. Larger values of $\Omega_{\mathrm{th}} h^{2}$ may be obtained by increasing $\mu$ which leads to reduced Higgsino component of the LSP, hence smaller annihilation cross-section. The LSP scattering cross-section on nucleons for all the benchmarks is below the LUX limits [68] but within the future reach of Xenon1T for direct detection via spin-independent (SI) LSP-nucleon interactions [69]. The compatibility with the LUX constraints is the reason for choosing negative value of $M_{1}$ in the benchmarks because $\mu M_{1}<0$ allows for some cancellations in the Higgs coupling to LSP which results in smaller SI scattering cross-section [70]. Additional suppression of the SI scattering cross-section may originate from destructive interference between the contributions to the SI scattering amplitude from the Higgs and the light singlet-scalar exchange which are of comparable size if Higgs-singlet mixing is non-negligible as in the present scenario [71]. For somewhat larger $\tan \beta$ SI scattering cross-section could be also suppressed by effects of destructive interference with the amplitude generated by MSSM-like Higgs exchange [72]. Due to small fraction of higgsino component of the LSP, hence small LSP coupling to Z boson, the spin-dependent (SD) scattering cross-section on neutrons is in the range between $10^{-7}$ and $10^{-6} \mathrm{pb}$ so about three order of magnitude below a recent LUX limit [73]. Limits from the SD scattering cross-section on protons are even weaker.

Since this scenario requires light highly-mixed stops and many additional light Higgs bosons there are non-negligible contributions to $B$-physics observables. We computed these observables with NMSSMTools that follows the calculation of ref. [74], which assumes Minimal Flavor Violation (MFV). For all benchmarks $\operatorname{BR}(b \rightarrow s \gamma), \operatorname{BR}\left(B_{s} \rightarrow \mu^{+} \mu^{-}\right)$and $\operatorname{BR}\left(B_{d} \rightarrow \mu^{+} \mu^{-}\right)$are below the $\mathrm{SM}$ prediction while $\operatorname{BR}\left(B \rightarrow X_{s} \mu^{+} \mu^{-}\right)$(in the low dimuon invariant mass region) is above the $\mathrm{SM}$ prediction. The $\mathrm{BR}(b \rightarrow s \gamma)$ is in agreement with the experimental central value [75] at the $2 \sigma$ level for all points apart from P2, while a similar discrepancy with the experiment for $\operatorname{BR}\left(B \rightarrow X_{s} \mu^{+} \mu^{-}\right)$is observed only for P1 and P2 [76]. This proves that it is possible to find points featuring th enhancement consistent with these observables, even under the Minimal Flavor Violation (MFV) assumption. It should be also emphasized that the predictions for $B$-physics observables 
are sensitive to sources of flavor violation beyond $\mathrm{MFV}$, for example to flavor structure of the down squark parameters via loops with gluinos [77], that have a minor effect in Higgs physics, that is the subject of this work. The only measurement that is in more than $2 \sigma$ tension with all the benchmarks in the MFV scenario is the combined CMS and LHCb analysis of $B_{s} \rightarrow \mu^{+} \mu^{-}$and $B_{d} \rightarrow \mu^{+} \mu^{-}$decays [78]. However, this is due to the fact that the SM prediction is already $2 \sigma$ away from the experimental central value. Moreover, in ref. [79] it was specifically demonstrated that $\operatorname{BR}\left(B_{s} \rightarrow \mu^{+} \mu^{-}\right)$and $\operatorname{BR}\left(B_{d} \rightarrow \mu^{+} \mu^{-}\right)$are very sensitive to non-MFV parameters, especially for low $\tan \beta$.

We should also warn the reader that benchmarks $\mathrm{P} 1-\mathrm{P} 4$ presented in table 1 possess unphysical global minimum characterised by vanishing $\langle S\rangle$ and $\left\langle H_{u}\right\rangle$ (or $\left\langle H_{d}\right\rangle$ ). Such minima have a depth $-2 m_{H_{d}}^{4} / g^{2}$ (or $-2 m_{H_{u}}^{4} / g^{2}$ ) and were analysed in the $\mathbb{Z}_{3}$-invariant NMSSM in refs. $[53,54,80]$. It is beyond the scope of the present paper to calculate the lifetime of the metastable EW vacuum for these benchmarks. However, in the general NMSSM there is enough freedom to make the EW vacuum deeper than the above mentioned unphysical minima while preserving characteristic features of benchmarks presented in table 1. For example, large negative $\xi_{S}$ can make the EW vacuum deeper than the unphysical ones. Such values of $\xi_{S}$ give positive contribution to the CP-even singlet mass but negative value of $\kappa A_{\kappa}$ can keep the singlet light. In table 2 we present benchmarks with the EW vacuum deeper than the unphysical ones. P5 has the same qualitative features as P1 except for heavy CP-odd singlet which results from large negative $\xi_{S}$. In consequence, only the CP-even singlet is present in decays of MSSM-like Higgs bosons, as seen from table 3. Nevertheless, the CP-odd singlet can be light also in this case for appropriately chosen positive value of $m_{S}^{\prime 2}$, as exemplified by benchmark P6.

\section{Conclusions}

The analysis of the Higgs precision measurement data at run I of the LHC have revealed large uncertainties in the coupling of the recently observed Higgs boson to the third generation quarks. In particular, the best fit value of the Higgs coupling to top quarks is more than $2 \sigma$ above the SM value. Similarly, the best fit of the Higgs coupling to bottom quarks is $2 \sigma$ below the SM value.

In this article we have investigated the possibility of obtaining such modifications of the third generation couplings within supersymmetric extensions of the Standard Model. In particular we have shown that they may be obtained in the NMSSM for values of the singlet state masses below $125 \mathrm{GeV}$ and values of the heavy CP-even Higgs mass of the order of a few hundred GeV. In addition, in order to get consistency with the observed gluon fusion rates, light stops and relatively large values of the stop mixing parameters are also required. We have discussed the phenomenological implications of this scenario, showing, for instance that it may be also compatible with the observed LEP2 excess in the search for associated production of Higgs bosons with neutral gauge bosons, $e^{+} e^{-} \rightarrow h Z$.

This scenario predicts not only deviations of the Higgs rates with respect to the SM values, but also a rich pattern of non-standard Higgs decays, many of which are not covered 
by existing LHC searches. It will be also tested in the search for light stops, and therefore may be probed in multiple channels at the LHC in the near future.

\section{Acknowledgments}

This work has been partially supported by National Science Centre under research grant DEC-2014/15/B/ST2/02157, by the Office of High Energy Physics of the U.S. Department of Energy under Contract DE-AC02-05CH11231, and by the National Science Foundation under grant PHY-1316783. MB acknowledges support from the Polish Ministry of Science and Higher Education (decision no. 1266/MOB/IV/2015/0). Work at the University of Chicago is supported in part by U.S. Department of Energy grant number DE-FG0213ER41958. Work at ANL is supported in part by the U.S. Department of Energy under Contract No. DE-AC02-06CH11357.

Open Access. This article is distributed under the terms of the Creative Commons Attribution License (CC-BY 4.0), which permits any use, distribution and reproduction in any medium, provided the original author(s) and source are credited.

\section{References}

[1] ATLAS collaboration, Observation of a new particle in the search for the Standard Model Higgs boson with the ATLAS detector at the LHC, Phys. Lett. B 716 (2012) 1 [arXiv: 1207.7214] [INSPIRE].

[2] CMS collaboration, Observation of a new boson at a mass of $125 \mathrm{GeV}$ with the CMS experiment at the LHC, Phys. Lett. B $\mathbf{7 1 6}$ (2012) 30 [arXiv:1207.7235] [INSPIRE].

[3] ATLAS and CMS collaborations, Measurements of the Higgs boson production and decay rates and constraints on its couplings from a combined ATLAS and CMS analysis of the LHC pp collision data at $\sqrt{s}=7$ and $8 \mathrm{TeV}$, JHEP 08 (2016) 045 [arXiv:1606.02266] [INSPIRE].

[4] ATLAS collaboration, Search for the associated production of the Higgs boson with a top quark pair in multilepton final states with the ATLAS detector, Phys. Lett. B 749 (2015) 519 [arXiv: 1506.05988] [INSPIRE].

[5] ATLAS collaboration, Search for $H \rightarrow \gamma \gamma$ produced in association with top quarks and constraints on the Yukawa coupling between the top quark and the Higgs boson using data taken at $7 \mathrm{TeV}$ and $8 \mathrm{TeV}$ with the ATLAS detector, Phys. Lett. B $\mathbf{7 4 0}$ (2015) 222 [arXiv: 1409.3122] [INSPIRE].

[6] CMS collaboration, Search for the associated production of the Higgs boson with a top-quark pair, JHEP 09 (2014) 087 [Erratum ibid. 10 (2014) 106] [arXiv:1408.1682] [INSPIRE].

[7] ATLAS collaboration, Search for the associated production of a Higgs boson and a top quark pair in multilepton final states with the ATLAS detector, ATLAS-CONF-2016-058, CERN, Geneva Switzerland, (2016).

[8] ATLAS collaboration, Combination of the searches for Higgs boson production in

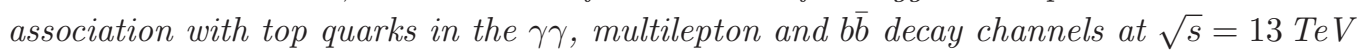
with the ATLAS detector, ATLAS-CONF-2016-068, CERN, Geneva Switzerland, (2016). 
[9] M. Badziak and C.E.M. Wagner, Enhancing the Higgs associated production with a top quark pair, JHEP 05 (2016) 123 [arXiv: 1602.06198] [INSPIRE].

[10] A. Azatov and A. Paul, Probing Higgs couplings with high $p_{T}$ Higgs production, JHEP 01 (2014) 014 [arXiv: 1309.5273] [INSPIRE].

[11] C. Grojean, E. Salvioni, M. Schlaffer and A. Weiler, Very boosted Higgs in gluon fusion, JHEP 05 (2014) 022 [arXiv: 1312.3317] [INSPIRE].

[12] S.P. Martin, A supersymmetry primer, Adv. Ser. Direct. High Energy Phys. 18 (1998) 1 [Adv. Ser. Direct. High Energy Phys. 21 (2010) 1] [hep-ph/9709356] [INSPIRE].

[13] U. Ellwanger, C. Hugonie and A.M. Teixeira, The next-to-minimal supersymmetric Standard Model, Phys. Rept. 496 (2010) 1 [arXiv:0910.1785] [InSPIRE].

[14] Delphi, OPAl, Aleph, LeP Working Group for Higgs Boson Searches and L3 collaborations, S. Schael et al., Search for neutral MSSM Higgs bosons at LEP, Eur. Phys. J. C 47 (2006) 547 [hep-ex/0602042] [InSPIRE].

[15] A. Angelescu, A. Djouadi and G. Moreau, Vector-like top/bottom quark partners and Higgs physics at the LHC, Eur. Phys. J. C 76 (2016) 99 [arXiv:1510.07527] [InSPIRE].

[16] CMS collaboration, Search for new physics in events with same-sign dileptons and jets in pp collisions at $\sqrt{s}=8 \mathrm{TeV}$, JHEP 01 (2014) 163 [Erratum ibid. 01 (2015) 014] [arXiv: 1311.6736] [INSPIRE].

[17] ATLAS collaboration, Search for supersymmetry at $\sqrt{s}=8 \mathrm{TeV}$ in final states with jets and two same-sign leptons or three leptons with the ATLAS detector, JHEP 06 (2014) 035 [arXiv: 1404.2500] [INSPIRE].

[18] ATLAS collaboration, Analysis of events with b-jets and a pair of leptons of the same charge in pp collisions at $\sqrt{s}=8 \mathrm{TeV}$ with the ATLAS detector, JHEP 10 (2015) 150 [arXiv: 1504.04605] [INSPIRE].

[19] P. Huang, A. Ismail, I. Low and C.E.M. Wagner, Same-sign dilepton excesses and light top squarks, Phys. Rev. D 92 (2015) 075035 [arXiv: 1507.01601] [INSPIRE].

[20] C.-R. Chen, H.-C. Cheng and I. Low, Same-sign dilepton excesses and vector-like quarks, JHEP 03 (2016) 098 [arXiv: 1511.01452] [INSPIRE].

[21] H.-C. Cheng, L. Li and Q. Qin, Second stop and sbottom searches with a stealth stop, JHEP 11 (2016) 181 [arXiv:1607.06547] [INSPIRE].

[22] D. Carmi, A. Falkowski, E. Kuflik, T. Volansky and J. Zupan, Higgs after the discovery: a status report, JHEP 10 (2012) 196 [arXiv:1207.1718] [INSPIRE].

[23] A. Djouadi, The anatomy of electro-weak symmetry breaking. II. The Higgs bosons in the minimal supersymmetric model, Phys. Rept. 459 (2008) 1 [hep-ph/0503173] [INSPIRE].

[24] ATLAS collaboration, Search for the supersymmetric partner of the top quark in the jets $+E_{\mathrm{T}}^{\mathrm{miss}}$ final state at $\sqrt{s}=13 \mathrm{TeV}$, ATLAS-CONF-2016-077, CERN, Geneva Switzerland, (2016).

[25] ATLAS collaboration, Search for top squarks in final states with one isolated lepton, jets and missing transverse momentum in $\sqrt{s}=13 \mathrm{TeV}$ pp collisions with the ATLAS detector, ATLAS-CONF-2016-050, CERN, Geneva Switzerland, (2016). 
[26] ATLAS collaboration, Search for direct top squark pair production and dark matter production in final states with two leptons in $\sqrt{s}=13 \mathrm{TeV}$ pp collisions using $13.3 \mathrm{fb}^{-1}$ of ATLAS data, ATLAS-CONF-2016-076, CERN, Geneva Switzerland, (2016).

[27] CMS collaboration, Search for direct top squark pair production in the fully hadronic final state in proton-proton collisions at $\sqrt{s}=13 \mathrm{TeV}$ corresponding to an integrated luminosity of $12.9 \mathrm{fb}^{-1}$, CMS-PAS-SUS-16-029, CERN, Geneva Switzerland, (2016).

[28] CMS collaboration, Search for direct top squark pair production in the single lepton final state at $\sqrt{s}=13 \mathrm{TeV}$, CMS-PAS-SUS-16-028, CERN, Geneva Switzerland, (2016).

[29] ATLAS collaboration, Search for new phenomena in final states with an energetic jet and large missing transverse momentum in pp collisions at $\sqrt{s}=13$ TeV using the ATLAS detector, Phys. Rev. D 94 (2016) 032005 [arXiv: 1604.07773] [InSPIRE].

[30] CMS collaboration, Search for direct production of bottom and light top squark pairs in proton-proton collisions at $\sqrt{s}=13 \mathrm{TeV}$, CMS-PAS-SUS-16-001, CERN, Geneva Switzerland, (2016).

[31] ATLAS collaboration, ATLAS run 1 searches for direct pair production of third-generation squarks at the Large Hadron Collider, Eur. Phys. J. C 75 (2015) 510 [Erratum ibid. C 76 (2016) 153] [arXiv:1506.08616] [INSPIRE].

[32] CMS collaboration, Search for top squark pair production in compressed-mass-spectrum scenarios in proton-proton collisions at $\sqrt{s}=8 \mathrm{TeV}$ using the $\alpha_{T}$ variable, submitted to Phys. Lett. B (2016) [arXiv: 1605.08993] [INSPIRE].

[33] M. Carena, I. Low, N.R. Shah and C.E.M. Wagner, Impersonating the Standard Model Higgs boson: alignment without decoupling, JHEP 04 (2014) 015 [arXiv: 1310.2248] [INSPIRE].

[34] M. Carena, H.E. Haber, I. Low, N.R. Shah and C.E.M. Wagner, Alignment limit of the NMSSM Higgs sector, Phys. Rev. D 93 (2016) 035013 [arXiv:1510.09137] [InSPIRE].

[35] K.S. Jeong, Y. Shoji and M. Yamaguchi, Singlet-doublet Higgs mixing and its implications on the Higgs mass in the PQ-NMSSM, JHEP 09 (2012) 007 [arXiv:1205.2486] [INSPIRE].

[36] K. Agashe, Y. Cui and R. Franceschini, Natural islands for a $125 \mathrm{GeV}$ Higgs in the scale-invariant NMSSM, JHEP 02 (2013) 031 [arXiv:1209.2115] [INSPIRE].

[37] M. Badziak, M. Olechowski and S. Pokorski, New regions in the NMSSM with a $125 \mathrm{GeV}$ Higgs, JHEP 06 (2013) 043 [arXiv: 1304.5437] [INSPIRE].

[38] U. Ellwanger, J.F. Gunion and C. Hugonie, NMHDECAY: a fortran code for the Higgs masses, couplings and decay widths in the NMSSM, JHEP 02 (2005) 066 [hep-ph/0406215] [INSPIRE].

[39] U. Ellwanger and C. Hugonie, NMHDECAY 2.0: an updated program for sparticle masses, Higgs masses, couplings and decay widths in the NMSSM, Comput. Phys. Commun. 175 (2006) 290 [hep-ph/0508022] [INSPIRE].

[40] P. Bechtle et al., HiggsBounds-4: improved tests of extended Higgs sectors against exclusion bounds from LEP, the Tevatron and the LHC, Eur. Phys. J. C 74 (2014) 2693 [arXiv:1311.0055] [INSPIRE].

[41] G. Bélanger, U. Ellwanger, J.F. Gunion, Y. Jiang, S. Kraml and J.H. Schwarz, Higgs bosons at 98 and $125 \mathrm{GeV}$ at LEP and the LHC, JHEP 01 (2013) 069 [arXiv:1210.1976] [INSPIRE]. 
[42] B. Bhattacherjee, M. Chakraborti, A. Chakraborty, U. Chattopadhyay, D. Das and D.K. Ghosh, Implications of the $98 \mathrm{GeV}$ and $125 \mathrm{GeV}$ Higgs scenarios in nondecoupling supersymmetry with updated ATLAS, CMS and Planck data, Phys. Rev. D 88 (2013) 035011 [arXiv: 1305.4020] [INSPIRE].

[43] U. Ellwanger and M. Rodriguez-Vazquez, Discovery prospects of a light scalar in the NMSSM, JHEP 02 (2016) 096 [arXiv: 1512.04281] [INSPIRE].

[44] B. Allanach, M. Badziak, C. Hugonie and R. Ziegler, Light sparticles from a light singlet in gauge mediation, Phys. Rev. D 92 (2015) 015006 [arXiv: 1502.05836] [InSPIRE].

[45] B.C. Allanach, M. Badziak, G. Cottin, N. Desai, C. Hugonie and R. Ziegler, Prompt signals and displaced vertices in sparticle searches for next-to-minimal gauge mediated supersymmetric models, Eur. Phys. J. C 76 (2016) 482 [arXiv:1606.03099] [INSPIRE].

[46] A. Delgado, G.F. Giudice and P. Slavich, Dynamical $\mu$ term in gauge mediation, Phys. Lett. B 653 (2007) 424 [arXiv:0706.3873] [InSPIRE].

[47] ATLAS collaboration, Measurement of fiducial, differential and production cross sections in the $H \rightarrow \gamma \gamma$ decay channel with $13.3 \mathrm{fb}^{-1}$ of $13 \mathrm{TeV}$ proton-proton collision data with the ATLAS detector, ATLAS-CONF-2016-067, CERN, Geneva Switzerland, (2016).

[48] CMS collaboration, Updated measurements of Higgs boson production in the diphoton decay channel at $\sqrt{s}=13 \mathrm{TeV}$ in pp collisions at CMS, CMS-PAS-HIG-16-020, CERN, Geneva Switzerland, (2016).

[49] J.A. Casas, A. Lleyda and C. Muñoz, Strong constraints on the parameter space of the MSSM from charge and color breaking minima, Nucl. Phys. B 471 (1996) 3 [hep-ph/9507294] [INSPIRE].

[50] D. Chowdhury, R.M. Godbole, K.A. Mohan and S.K. Vempati, Charge and color breaking constraints in MSSM after the Higgs discovery at LHC, JHEP 02 (2014) 110 [arXiv:1310.1932] [INSPIRE].

[51] N. Blinov and D.E. Morrissey, Vacuum stability and the MSSM Higgs mass, JHEP 03 (2014) 106 [arXiv:1310.4174] [INSPIRE].

[52] J.E. Camargo-Molina, B. Garbrecht, B. O'Leary, W. Porod and F. Staub, Constraining the natural MSSM through tunneling to color-breaking vacua at zero and non-zero temperature, Phys. Lett. B 737 (2014) 156 [arXiv:1405.7376] [INSPIRE].

[53] Y. Kanehata, T. Kobayashi, Y. Konishi, O. Seto and T. Shimomura, Constraints from unrealistic vacua in the next-to-minimal supersymmetric Standard Model, Prog. Theor. Phys. 126 (2011) 1051 [arXiv:1103.5109] [INSPIRE].

[54] T. Kobayashi, T. Shimomura and T. Takahashi, Constraining the Higgs sector from false vacua in the next-to-minimal supersymmetric Standard Model, Phys. Rev. D 86 (2012) 015029 [arXiv:1203.4328] [InSPIRE].

[55] U. Ellwanger and C. Hugonie, Constraints from charge and color breaking minima in the (M+1)SSM, Phys. Lett. B 457 (1999) 299 [hep-ph/9902401] [InSPIRE].

[56] R.V. Harlander, S. Liebler and H. Mantler, SusHi: a program for the calculation of Higgs production in gluon fusion and bottom-quark annihilation in the Standard Model and the MSSM, Comput. Phys. Commun. 184 (2013) 1605 [arXiv: 1212.3249] [INSPIRE].

[57] R.V. Harlander, S. Liebler and H. Mantler, SusHi Bento: beyond NNLO and the heavy-top limit, Comput. Phys. Commun. 212 (2017) 239 [arXiv:1605.03190] [INSPIRE]. 
[58] CMS collaboration, Search for $H / A$ decaying into $Z+A / H$, with $Z \rightarrow \ell \ell$ and $A / H$ to fermion pair, CMS-PAS-HIG-15-001, CERN, Geneva Switzerland, (2015).

[59] CMS collaboration, Search for $H \rightarrow Z(\ell \ell)+A(b \bar{b})$ with 2015 data, CMS-PAS-HIG-16-010, CERN, Geneva Switzerland, (2016).

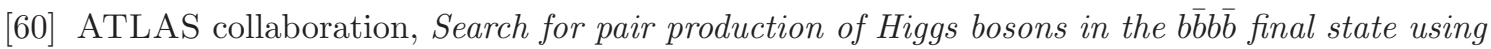
proton-proton collisions at $\sqrt{s}=13 \mathrm{TeV}$ with the ATLAS detector,

Phys. Rev. D 94 (2016) 052002 [arXiv: 1606.04782] [inSPIRE].

[61] CMS collaboration, Search for resonant pair production of Higgs bosons decaying to two bottom quark-antiquark pairs in proton-proton collisions at $13 \mathrm{TeV}$, CMS-PAS-HIG-16-002, CERN, Geneva Switzerland, (2016).

[62] ATLAS collaboration, Search for charged Higgs bosons in the $H^{ \pm} \rightarrow t b$ decay channel in $p p$ collisions at $\sqrt{s}=13 \mathrm{TeV}$ using the ATLAS detector, ATLAS-CONF-2016-089, CERN, Geneva Switzerland, (2016).

[63] C. Degrande, R. Frederix, V. Hirschi, M. Ubiali, M. Wiesemann and M. Zaro, Accurate predictions for charged Higgs production: closing the $m_{H^{ \pm}} \sim m_{t}$ window, arXiv:1607.05291 [INSPIRE].

[64] P. Bandyopadhyay, K. Huitu and S. Niyogi, Non-standard charged Higgs decay at the LHC in next-to-minimal supersymmetric Standard Model, JHEP 07 (2016) 015 [arXiv:1512.09241] [INSPIRE].

[65] CMS collaboration, Search for a neutral MSSM Higgs boson decaying into $\tau \tau$ at $13 \mathrm{TeV}$, CMS-PAS-HIG-16-006, CERN, Geneva Switzerland, (2016).

[66] G. Bélanger, F. Boudjema, A. Pukhov and A. Semenov, MicrOMEGAs3: a program for calculating dark matter observables, Comput. Phys. Commun. 185 (2014) 960 [arXiv: 1305.0237] [INSPIRE].

[67] Planck collaboration, P.A.R. Ade et al., Planck 2015 results. XIII. Cosmological parameters, Astron. Astrophys. 594 (2016) A13 [arXiv:1502.01589] [INSPIRE].

[68] LUX collaboration, D.S. Akerib et al., Results from a search for dark matter in the complete LUX exposure, Phys. Rev. Lett. 118 (2017) 021303 [arXiv: 1608.07648] [INSPIRE].

[69] XENON collaboration, E. Aprile et al., Physics reach of the XENON1T dark matter experiment, JCAP 04 (2016) 027 [arXiv: 1512.07501] [INSPIRE].

[70] C. Cheung, L.J. Hall, D. Pinner and J.T. Ruderman, Prospects and blind spots for neutralino dark matter, JHEP 05 (2013) 100 [arXiv:1211.4873] [INSPIRE].

[71] M. Badziak, M. Olechowski and P. Szczerbiak, Blind spots for neutralino dark matter in the NMSSM, JHEP 03 (2016) 179 [arXiv:1512.02472] [INSPIRE].

[72] P. Huang and C.E.M. Wagner, Blind spots for neutralino dark matter in the MSSM with an intermediate $m_{A}$, Phys. Rev. D 90 (2014) 015018 [arXiv: 1404.0392] [INSPIRE].

[73] LUX collaboration, D.S. Akerib et al., Results on the spin-dependent scattering of weakly interacting massive particles on nucleons from the run 3 data of the LUX experiment, Phys. Rev. Lett. 116 (2016) 161302 [arXiv:1602.03489] [INSPIRE].

[74] F. Domingo, Update of the flavour-physics constraints in the NMSSM, Eur. Phys. J. C 76 (2016) 452 [arXiv:1512.02091] [INSPIRE]. 
[75] Heavy Flavor Averaging Group (HFAG) collaboration, Y. Amhis et al., Averages of b-hadron, c-hadron and $\tau$-lepton properties as of summer 2014, arXiv:1412.7515 [INSPIRE].

[76] T. Huber, T. Hurth and E. Lunghi, Inclusive $\bar{B} \rightarrow X_{s} \ell^{+} \ell^{-}$: complete angular analysis and a thorough study of collinear photons, JHEP 06 (2015) 176 [arXiv: 1503.04849] [INSPIRE].

[77] F. Gabbiani, E. Gabrielli, A. Masiero and L. Silvestrini, A complete analysis of FCNC and CP constraints in general SUSY extensions of the Standard Model, Nucl. Phys. B 477 (1996) 321 [hep-ph/9604387] [INSPIRE].

[78] LHCb and CMS collaborations, Observation of the rare $B_{s}^{0} \rightarrow \mu^{+} \mu^{-}$decay from the combined analysis of CMS and LHCb data, Nature $\mathbf{5 2 2}$ (2015) 68 [arXiv:1411.4413] [INSPIRE].

[79] A. Dedes, J. Rosiek and P. Tanedo, Complete one-loop MSSM predictions for $B^{0} \rightarrow \ell^{+} \ell^{\prime-}$ at the Tevatron and LHC, Phys. Rev. D 79 (2009) 055006 [arXiv:0812.4320] [InSPIRE].

[80] U. Ellwanger, M. Rausch de Traubenberg and C.A. Savoy, Phenomenology of supersymmetric models with a singlet, Nucl. Phys. B 492 (1997) 21 [hep-ph/9611251] [INSPIRE]. 